Proc. of the 15th Int. Workshop on Slow Positron Beam Techniques and Applications, Prague, September 2-6, 2019

\title{
Development of J-PEM for Breast Cancer Detection
}

\author{
SHIVANI $^{a, *}$, E. EUCZYŃSKA ${ }^{b}$, S. HEINZE ${ }^{c}$ AND P. MOSKAL ${ }^{a}$ \\ ${ }^{a}$ Faculty of Physics and Applied Computer Science, Jagiellonian University, Cracow, Poland \\ ${ }^{b}$ Medical College of Rzeszow University, Cracow, Poland \\ ${ }^{c}$ Department of Radiology, Center of Oncology, Cracow Branch, Cracow, Poland
}

\begin{abstract}
A detection system of the conventional PET tomograph is set-up to record data from $e^{+} e^{-}$annihilation into two photons, each with energy of $511 \mathrm{keV}$, and to give information about the spatial density distribution of a radiopharmaceutical in the patients body. Dedicated positron emission mammography (PEM) systems provide a potentially high sensitivity, high-resolution, low attenuation, and lower cost alternative to whole body PET. We have designed, built, and performed initial evaluation of a large field-of-view Jagiellonian Positron Emission Mammography (J-PEM) system. This 3D system is based on novel idea of applying plastic scintillators to detect annihilation photons and improving spatial resolution by utilization of wavelength shifters (WLS). In addition, this device is being developed in view of classification of malignancy based on the possibility of positronium mean lifetime imaging. Here we present the first results from the simulations as motivation for our investigation.
\end{abstract}

DOI: 10.12693/APhysPolA.137.140

PACS/topics: 78.70.Bj, 41.75.Fr, 36.10.Dr

\section{Introduction}

Dedicated detectors for imaging positron-emitting tracers in the breast have several benefits compared to whole-body tomographs, including high sensitivity for the emitted radiation, much lower attenuation, and potentially much lower cost [1]. One approach to positron emission mammography (PEM) is to use two planar detectors, above and below a compressed breast [2]. As with whole-body systems, several factors affect design of PEM systems, including the desire for a large enough field of view to image most breast sizes, high spatial resolution, high coincidence detection efficiency, good energy resolution (for scatter rejection), high countrate capability, and good timing (for random event rejection). Our aim is to improve sensitivity and spatial resolution by means of new techniques utilizing plastic scintillators $[3,4]$ and wavelength shifters [5]. Additionally this device will be developed in view of classification of malignancy based on the possibility of positronium mean lifetime imaging $[6,7]$.

The aim of this article is to present the characteristics response of the J-PEM scanner based on simulations and estimating the point spread function (PSF) and sensitivity of the detector. The above characteristics may be used as a figure of merit in the geometry optimization of the prototype device. Because of the fact that the J-PEM scanner is meant to be used in medical diagnostics, the target performance should be at least comparable to the performance of currently available commercial PEM devices. The concept of J-PEM

\footnotetext{
* corresponding author; e-mail:

shivani.shivani@doctoral.uj.edu.pl
}

scanner is discussed in Sect. 2. Section 3 is dedicated to Monte Carlo simulations with the uses of which an annihilation point is determined. Conclusions are given in Sect. 4.

\section{Concept of J-PEM}

Positron Emission Mammography(PEM) is one of the most widespread modalities currently available for diagnosing breast cancers [2]. The main benefits of PEM include higher spatial resolution, improved sensitivity with reduced attenuation, shorter imaging time, and the possibility of reducing the radiopharmaceutical dose compared to whole-body PET. However, the precise measurement of photon energies requires applications of high resolution semiconductor detectors [8]. We propose to build a PEM with new techniques based on the concept of using plastic scintillator strips and Wavelength shifters [9], called J-PEM. The J-PEM is a prototype, which intended to evaluate PET technology in the diagnosis of malign neoplasm in the breast and of ganglion loco-regional invasion [10]. It is based on plastic scintillators and utilizes the same technology as the Jagiellonian Positron Emmision Tomograph, J-PET [11]. It is optimized for the detection of photons from electronpositron annihilation $[3,4]$. Such photons, having an energy of $511 \mathrm{keV}$, interact with electrons in plastic scintillators predominantly via the Compton effect. J-PEM uses a dedicated instrument for breast cancer detection that is equipped with two parallel photon detectors in a configuration similar to mammography compressors. The detector system consists of two modules of plastic scintillators, with each module built from two layers of plastic scintillator and the wavelength shifters [5,9] placed orthogonally between them, as shown in Fig. 1. Each scintillator bar is attached at both ends with Silicon Photomultipliers for the signal readout [3]. 

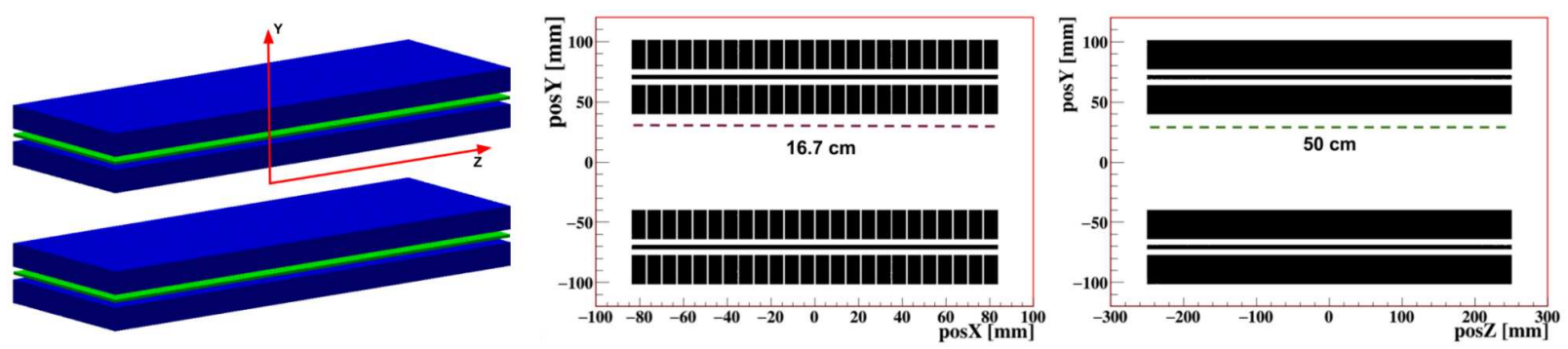

Fig. 1. Left: Geometry scheme of J-PEM used in GATE simulation. Blue color represents plastic scintillators and green color represents wavelength shifters. Dimension of plastic scintillators and WLS used are equal to $6 \times 24 \times 500 \mathrm{~mm}^{3}$ and $3 \times 10 \times 167 \mathrm{~mm}^{3}$, respectively. Space between the modules amounts to $8 \mathrm{~cm}$. The Y-Z (middle) and the X-Y (right) plane projection of the given geometry.

(a)

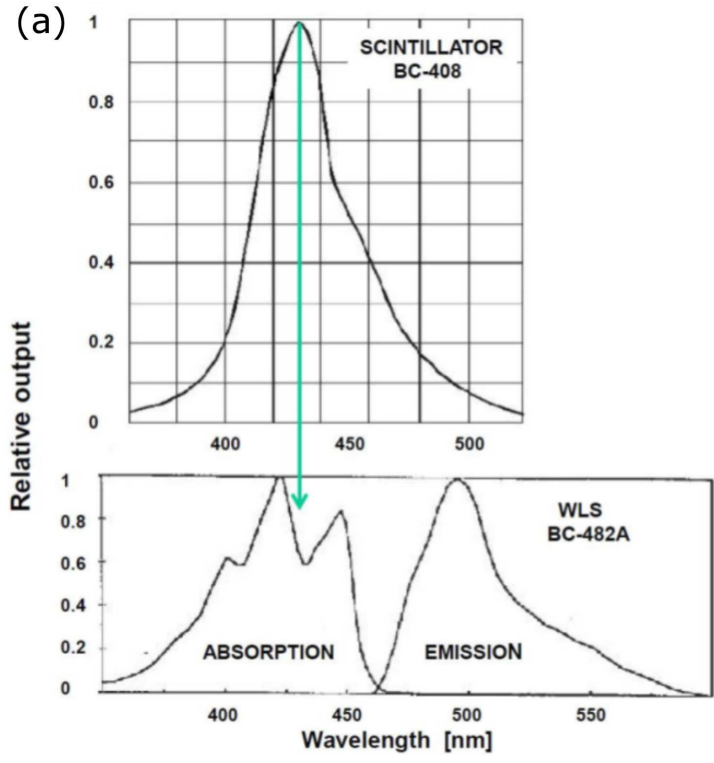

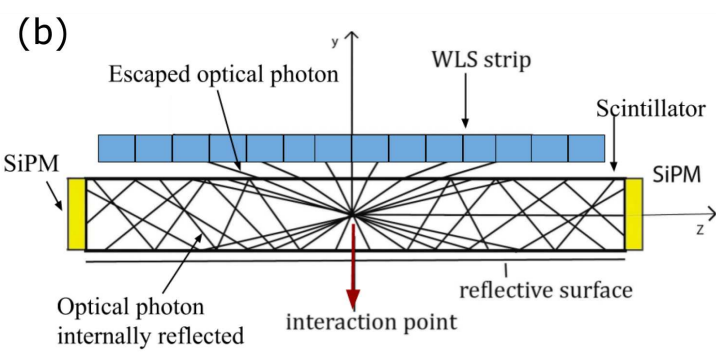

(c)

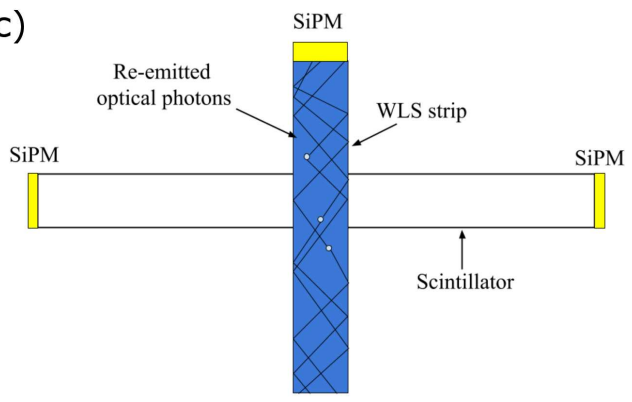

Fig. 2. (a) The emission and absorption spectra for exemplary plastic scintillator and WLS. (b) The propagation of the scintillation photons in the strip. The trajectories of photons emitted from the interaction point placed in the geometrical center are indicated by thick black lines [13]. (c) The illustrate re-emission of photons inside the WLS propagating towards the SiPM. White dots are the representation of number of absorbed scintillation photons inside WLS strip.

In the J-PET, the position of the interaction point of gamma quanta in plastic scintillators are calculated based on the measurement of the time difference of the signal arrivals to the ends of strips [3, 12]. Plastic scintillators provide time resolutions which results in accuracy of determination of annihilation point of around $10 \mathrm{~mm}$ [3]. That resolution can be improved by registering scintillation light escaping the scintillator bar through a side wall using a set of parallel wavelength-shifting (WLS) strips.

Some photons in the scintillator strip can be emitted at the angle lower than the critical angle, which makes them escape the scintillator bar instead of undergoing total internal reflection [5]. If the emission spectrum of scintillator is overlapping with the absorption spectrum of the WLS then those photons can be absorbed in
WLS strips. Next WLS is re-emitting isotropically photons with the wavelength shifted towards larger values. These photons can be registered by SiPM at the end of the WLS, as shown in Fig.2.

The coordinate of the interaction point along the scintillator strip (Z-axis) is determined as a weighted average of Z-coordinates of WLS strips, with weights equal to the amplitudes of signals registered in the WLS strips being proportional to the number of absorbed scintillation photons [9]. It has been already proven that one can achieve the position resolution of $5 \mathrm{~mm}(\sigma)$ for the coordinate along the scintillator bar by using WLS strips [9]. Here we discuss the design of J-PEM which consist of 24 plastic scintillator and 49 WLS in one module, with dimensions $6 \times 24 \times 500 \mathrm{~mm}^{3}$ and $3 \times 10 \times 167 \mathrm{~mm}^{3}$, respectively. 


\section{Monte Carlo Simulation}

In order to quantify the J-PEM geometry we have performed Monte-Carlo simulations using the GATE package, a platform developed by the Open-GATE collaboration [14] based on Geant4 software. It is dedicated to numerical simulations in medical imaging and radiotherapy. In the simulations the full geometry of the J-PEM detector and the composition of the detector material were taken into account. The interactions of photons in the scintillators were simulated by GATE. In the simulations we assumed that annihilation source is placed in the center of the detector and that the backto-back photons (each with an energy of $511 \mathrm{keV}$ ) from the $e^{+} e^{-} \rightarrow 2 \gamma$ annihilation are isotropically emitted. For analysis we are only accepting events with exactly two interactions and select events for which energy loss is larger than a given threshold value. Here we study two threshold values of $100 \mathrm{keV}$ and $200 \mathrm{keV}$. The latter value is anticipated for the reduction of the scatter fraction in the total-body PET case [12]. In case of J-PEM, however, we have anticipated that the expected scatter fraction will be smaller, and therefor a study also with the threshold of $100 \mathrm{keV}$ is performed.

\subsection{Determination of annihilation point}

The aim of J-PEM is to reconstruct the point of annihilation along the line of response (LOR) by calculating the time difference between two interactions.

If the two annihilation photons pass through the body without any scattering and can be detected in coincidence, a true LOR can be formed which includes the point of annihilation. Annihilation point of interaction denoted as $\boldsymbol{X}_{a}=\left(X_{a}, Y_{a}, Z_{a}\right)$ is calculated as:

$$
\boldsymbol{X}_{a}=\boldsymbol{X}_{0}+\frac{1}{2} c \Delta t \cdot \widehat{v}
$$

where $\boldsymbol{X}_{0}=\left(X_{0}, Y_{0}, Z_{0}\right)$ is vector pointing towards the middle of LOR, $\widehat{v}$ is a unit vector along LOR, $\Delta t$ is the time of flight and $c$ is the speed of light. $\boldsymbol{X}_{0}$ can be calculated as:

$$
\boldsymbol{X}_{0}=\frac{\boldsymbol{X}_{1}+\boldsymbol{X}_{2}}{2}
$$

where $\boldsymbol{X}_{1}$ and $\boldsymbol{X}_{2}$ are the position vector of two hits. The actual position of annihilation spot along LOR can be inferred using

$$
\Delta t=\left(t_{2}-t_{1}\right),
$$

where $t_{2}$ and $t_{1}$ are the hit time of the interactions. The hit position and time $\left(X_{i}, Y_{i}, Z_{i}, t_{i}\right)$ is pictorially shown in Fig. 3.

The simulations were performed for isotropic emission of back to back gamma from point like source placed in the centre of the detector. From the preliminary measurement of the scintillating module mentioned in the article, we were able to obtain the resolution of time and position along the $\mathrm{Z}$ axis. Since we are not able to determine the $\mathrm{X}$ and $\mathrm{Y}$ position of interaction, we are assuming that it occurred in the center of the scintillator. Preliminary resolution was $\sigma_{t}=60 \mathrm{~ns}$ and

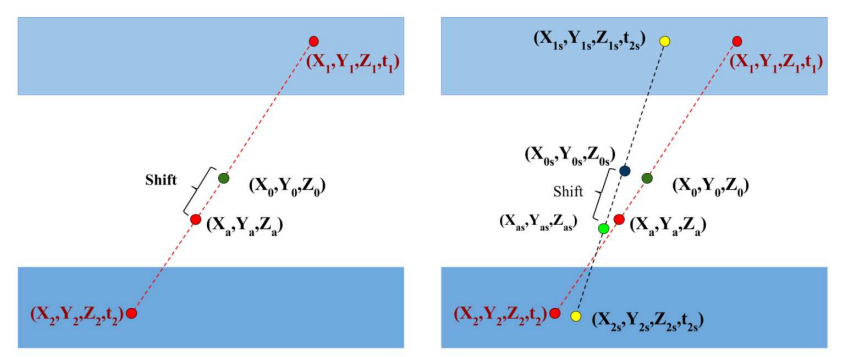

Fig. 3. Illustration of the formation of a LOR. Left: The pictorial representation of estimation of annihilation point, where $\boldsymbol{X}_{0}$ is the vector representing the middle of LOR, $\boldsymbol{X}_{a}$ is the annihilation point vector calculated using (1). The $\left(X_{i}, Y_{i}, Z_{i}, t_{i}\right)$ is the hit position and time of interaction of back to back gamma. Right: The $\boldsymbol{X}_{s}$ denotes interaction points after applying smearing simulating experimental resolution.

$\sigma_{\mathrm{Z}}=1.28 \mathrm{~mm}$, which this is the standard deviation of the Gaussian distribution. It can be related to FWHM of that Gaussian by the FWHM $\approx 2.355 \sigma$. In order to take into account the experimental spatial and time resolution, we smear the values of $t$ and $\mathrm{X}$ using Gaussian distribution with $\sigma_{t}=60 \mathrm{ps}$ and $\sigma_{\mathrm{Z}}=1.28 \mathrm{~mm}$, respectively. We assume that coincidence resolving time (CRT) is equal to $200 \mathrm{ps}\left(\sigma_{t}=60 \mathrm{ps}\right)$ and for $\mathrm{Z}$ direction FWHM is equal to $3 \mathrm{~mm}\left(\sigma_{\mathrm{Z}}=1.28 \mathrm{~mm}\right)$. To extract true coincidences from the set of all coincidences only events with exactly two interactions, registered with an energy loss larger than $200 \mathrm{keV}$, are considered. Such threshold suppresses scattering in the body to the level of about 35\% [15], and reduces to a negligible level that coincidences inside the detector. This is because the $511 \mathrm{keV}$ gamma quanta cannot deposit more than $184 \mathrm{keV}$ in more than one scattering [15]. As J-PEM is dedicated for breast imaging, we expect lower scatter fraction than the standard PET scanner. For this reason we have studied also a case with energy loss threshold of $100 \mathrm{keV}$.

In this article, we focus on estimation of the spatial resolution, and sensitivity as a main characteristics of detector performance. Point spread function (PSF) is a measure of spatial resolution of the scanner. It is obtained by applying the reconstruction procedure to the point like source [16]. This parameter is important for determining the suitability of an imaging system and for the possible restoration of fine details in images. PSF is calculated as the width at half of maximum, of a reconstructed point distribution, along each axis. For the calculations we are taking a slice of the reconstructed $X, Y$, and $\mathrm{Z}$ distribution of the annihilation point in the range of $(-0.5: 0.5) \mathrm{mm}$ around the position of the source. The $\mathrm{X}$ and $\mathrm{Y}$ positions of the interaction points are assumed to be in the center of the scintillators, so the distribution of LORs is discrete in $\mathrm{X}$ and $\mathrm{Y}$ dimension of the reconstructed image, as shown in Fig. 4. We have simulated $10^{7}$ events in total. The results for the distribution of annihilation point and their projections are shown in Fig. 4. 

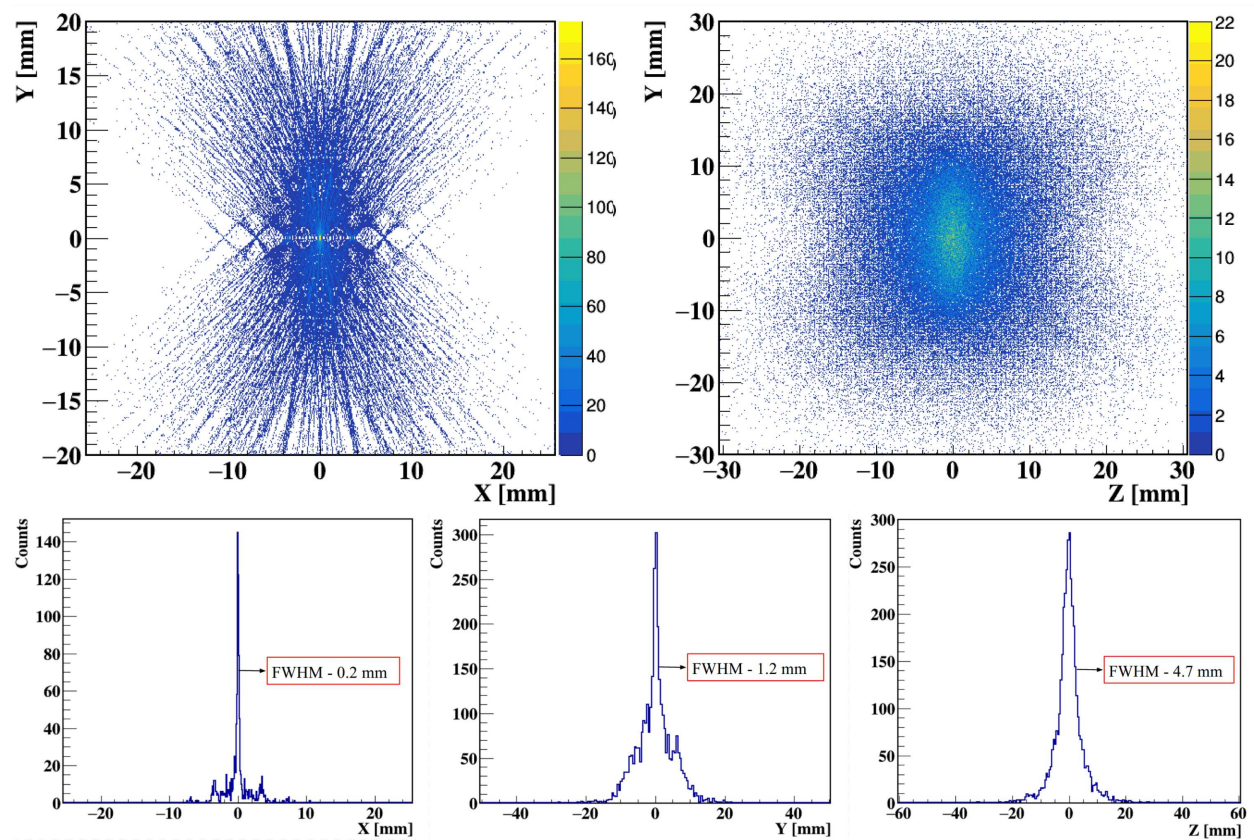

Fig. 4. Top plots show the distribution of annihilation point in the XY and the ZY plane. Bottom plots indicate the projection of $\mathrm{X}, \mathrm{Y}$, and $\mathrm{Z}$, performed for the cross section including the highest counting bin.
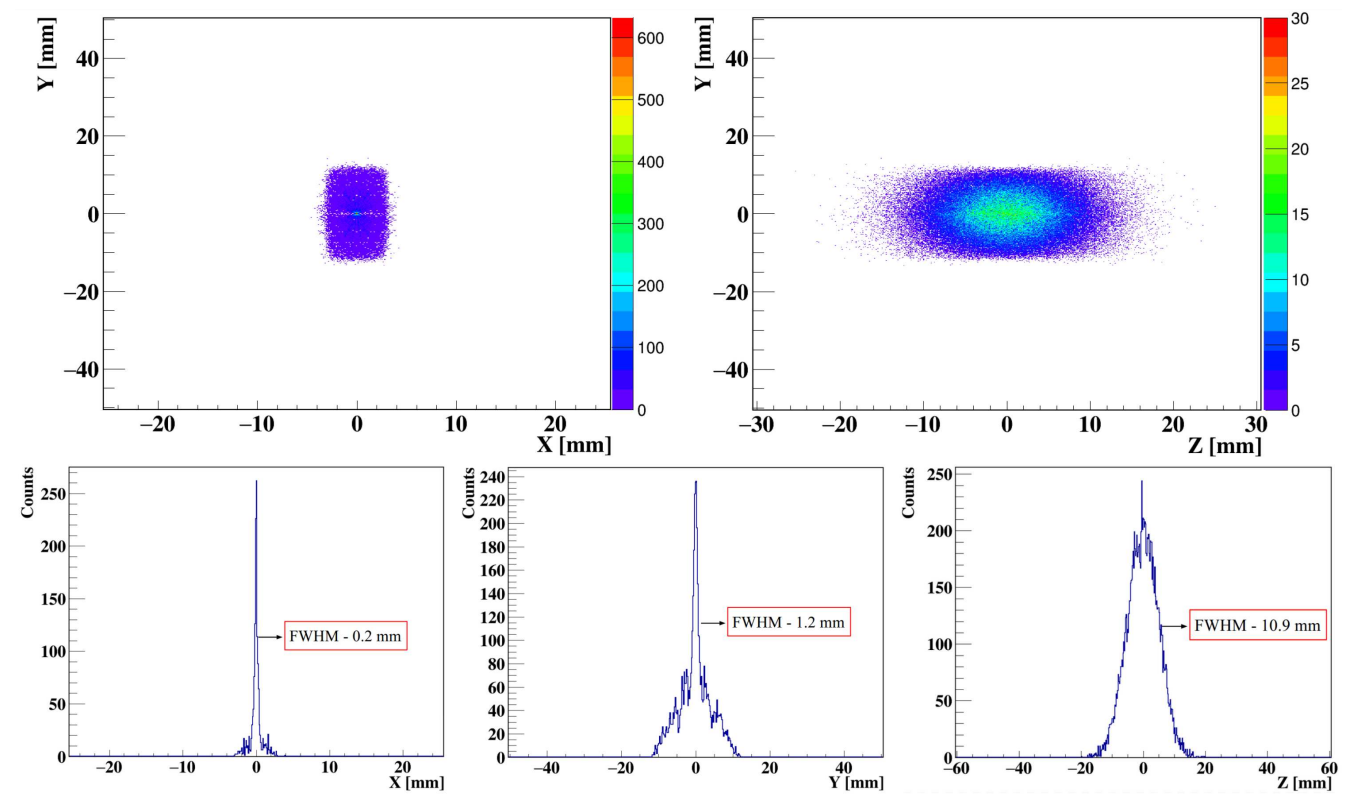

Fig. 5. The distribution of annihilation point in the XY and the ZY plane (top) and the projection of X, Y, and Z, performed for the cross section including the highest counting bin without WLS strips (bottom).

Based on the projection we have calculated that the values of PSF for X, Y, and $\mathrm{Z}$ are $0.2 \mathrm{~mm}, 1.2 \mathrm{~mm}$, and $4.7 \mathrm{~mm}$, respectively. Note that these values are different which might be due to asymmetry of the detector geometry, as previously mentioned. To make a comparison we have done the simulations without the WLS strips with $\sigma_{\mathrm{Z}}=10 \mathrm{~mm}$, and $\sigma_{t}=0 \mathrm{~ns}$ with the energy loss threshold of $200 \mathrm{keV}$ without WLS strips. The values of PSF for $\mathrm{X}, \mathrm{Y}$, and $\mathrm{Z}$ are $0.1 \mathrm{~mm}, 1.2 \mathrm{~mm}$ and $10.9 \mathrm{~mm}$, respectively, as shown in Fig. 5. This study demonstrates that the value of the PSF for the $\mathrm{Z}$ axis obtained with the WLS is equal to $4.7 \mathrm{~mm}$, whereas without the WLS the PSF value equals $10.9 \mathrm{~mm}$. It is clear now that the use of the WLS strips reduces the value of PSF along Z-direction to the half. This also means that the resolution becomes twice as good. 
TABLE I

Sensitivity of different PET scanners [17, 18] along with the J-PEM estimated in this article for two different energy loss thresholds.

\begin{tabular}{l|c|c|c}
\hline \hline \multicolumn{1}{c|}{ Scanners } & Sensitivity & $\begin{array}{c}\text { Detector ring } \\
\text { diameter }[\mathrm{cm}]\end{array}$ & $\begin{array}{c}\text { Crystal } \\
\text { size }\left[\mathrm{mm}^{3}\right]\end{array}$ \\
\hline D-IQ-5 & 22.8 & 74 & $6.3 \times 6.3 \times 30$ \\
D-600 & 9.4 & 80.1 & $4.7 \times 6.3 \times 30$ \\
D-STE & 8.8 & 88.6 & $4.7 \times 6.3 \times 30$ \\
D-ST & 8.7 & 88.6 & $6.3 \times 6.3 \times 30$ \\
J-PET & 14.9 & 85 & $7 \times 500 \times 19$ \\
J-PEM (200 keV) & 20.0 & 8 & $6 \times 500 \times 24$ \\
J-PEM (100 keV) & 31.4 & 8 & $6 \times 500 \times 24$
\end{tabular}

The sensitivity of a positron emission tomograph is expressed as the true coincidence events rate $T$, normalized to the total activity $A$ of the source. As per definition, we have calculated the sensitivity for J-PEM scanner, which occurs to be equal $20.0 \mathrm{cps} / \mathrm{kBq}$ and $31.4 \mathrm{cps} / \mathrm{kBq}$ for $200 \mathrm{keV}$ and $100 \mathrm{keV}$ energy threshold, respectively. Typically the distance between the detectors can range from 5 to $10 \mathrm{~cm}$, depending on the breast compression used [19]. Here we use the distance of $8 \mathrm{~cm}$ which is close to average used. In Table I the J-PEM sensitivity is compared to sensitivities of the standard PET scanners.

\section{Conclusion}

In this paper we have investigated the design and establishment of the characteristic performance of the Jagiellonian Positron Emission Mammography (J-PEM) for the detection and diagnosis of breast cancer. This construction is based on a novel idea of PET based on plastic scintillators and wavelength shifter (WLS) and a new concept of positronium imaging. We have prepared a simulation program based on Monte Carlo method using GATE toolkit, for optimizing the geometry and material for the J-PEM prototype. We have also presented the first results from the simulations. Results from the simulations are showing that the estimated sensitivity of J-PEM is higher than the other existing scanners, as shown in Table I. Simulations have been performed for two cases: (i) with WLS strips $\left(\sigma_{\mathrm{Z}}=1.28 \mathrm{~mm}\right)$ and (ii) without WLS strips $\left(\sigma_{\mathrm{Z}}=10 \mathrm{~mm}\right)$, to comprehend the difference. One can clearly estimate that using WLS strips reduced the value of PSF along Z-direction to the almost half $(4.7 \mathrm{~mm})$. This means that the resolution improves twice.

\section{References}

[1] E. Łuczyńska, S. Heinze-Paluchowska, S. Dyczek, P. Blecharz, J. Rys, M. Reinfuss, Korean J. Radiol. 15, 689 (2014).

[2] C. J. Thompson, K. Murthy, I.N. Weinberg, F. Mako, Medical Physics 21, 529 (1994).

[3] P. Moskal, Sz. Niedźwiecki, T. Bednarski, et al., Nucl. Inst. Meth. A 764, 317 (2014).

[4] P. Moskal, O. Rundel, D. Alfs, et al., Phys. Med. Biol. 61, 2025 (2016).

[5] J. Smyrski, P. Moskal, T. Bednarski et al., BioAlgorithms and Med-Systems 10, 59 (2014).

[6] P. Moskal, D. Kisielewska, C. Curceanu, et al., Phys. Med. Biol. 64, 055017 (2019).

[7] P. Moskal, B. Jasińska, E.Ł. Stępień and S.D. Bass, Nat. Rev. Phys. 1, 527 (2019).

[8] E. Abuelhia, K. Alzimami, M. Alkhorayef, Z. Podolyák, N.M. Spyrou, JRNC 278, 767 (2008).

[9] J. Smyrski, D. Alfs, T. Bednarski, et al., Nucl. Instr. Meth. Phys. Res. A 851, 39 (2017).

[10] Wen-Hu Hsu, Po-Kuei Hsu, Shyh-Jen Wang, Ko-Han Lin, Chien-Sheng Huang, Chih-Cheng Hsieh, Yu-Chung Wu, Ann Thorac. Surg. 87, 1564 (2009).

[11] Shivani, E. Łuczyńskab, S. Heinzec, P. Moskal, Acta Phys. Pol. B 51, 281 (2020).

[12] Sz. Niedźwiecki, P. Białas, C. Curceanu et al., Acta Phys. Pol. B 48, 1567 (2017).

[13] Shivani, DAE Symp. Nucl. Phys. 63, 1092 (2018).

[14] S. Jan, G. Santin, D. Strul, et al. Phys. Med. Biol. 49, 4543 (2004).

[15] P. Kowalski, W. Wiślicki, L. Raczyński, et al. Acta Phys. Pol. B 47, 549 (2016).

[16] G. Dougherty, Z. Kawaf, Radiography 7, 255 (2001).

[17] E. De Ponti, S. Morzenti, L. Guerra, C. Pasquali, M. Arosio, V. Bettinardi, A. Crespi, M.C. Gilardi, C. Messa, Med Phys. 38(2), 968 (2011).

[18] C. Gámez-Cenzano, I. Romero-Zayas, L. RodríguezBel, J.L. Vercher-Conejero, J.M. Martí-Climent, J. Nucl. Med. 58, 1155 (2017).

[19] M.V. Martins, Positron Emission Mammography in: Mammography Techniques and Review, Eds. F. Fernandes, L. Brasil, R. Guadagnin, IntechOpen, 2015. 